\title{
A Low-Loss Split-Carrier Transmitter Architecture for Intra-Datacentre Communications
}

\author{
Thomas Gerard(1), Sezer Erkılınç(1), Zhixin Liu( ${ }^{(1)}$, Benn Thomsen(2), Polina Bayvel(1), Domaniç Lavery(1) \\ (1) Optical Networks Group, Department of Electronic \& Electrical Engineering, University College Lon- \\ don, Torrington Place, London, UK, WC1E 7JE, uceetmh@ucl.ac.uk \\ (2) Microsoft Research Cambridge, 21 Station Rd, Cambridge, UK, CB1 2FB.
}

\begin{abstract}
A split-carrier transmitter is proposed to increase the loss budget for intra-datacentre links by reducing modulator insertion loss. Transmission of $28 \mathrm{GBd}$ subcarrier modulated 16-QAM (112 Gb/s) over $2 \mathrm{~km} \mathrm{SSMF}$ is shown to be possible with an optimised modulator loss of $<4.4 \mathrm{~dB}$.
\end{abstract}

\section{Introduction}

To meet the growing bandwidth requirements of cloud data services, datacentre intraconnects will be inevitably upgraded from 100 to $400 \mathrm{~Gb} / \mathrm{s}$. To achieve this, $56 \mathrm{GBd}$ 4-level pulse amplitude modulation (4PAM) using four wavelengths or fibres has been favoured as a low cost, low complexity solution ${ }^{1}$. However, 4PAM offers a relatively low loss budget, leaving little margin for system variation and future data rate improvements. The loss budget determines the continuous wave (CW) laser power required, defined here as

$$
P_{\mathrm{CW}, \text { req }}=P_{\mathrm{mod}, \mathrm{loss}}+P_{\mathrm{fibre}, \mathrm{loss}}+P_{\mathrm{Rx}, \mathrm{req}},
$$

where $P_{\text {mod,loss }}$ is the dynamic modulator loss in $\mathrm{dB}, P_{\text {fibre,loss }}$ is the fibre transmission loss in $\mathrm{dB}$, and $P_{\mathrm{Rx}, \text { req }}$ is the power required at the receiver, in $\mathrm{dBm}$, for a specified bit error rate (BER).

The CW launch power should be kept as low as possible to minimise the equipment and operational costs. To realise this, reduction of $P_{\text {mod,loss }}$ has been demonstrated using direct modulation, but requires nonlinear Volterra equalisation ${ }^{2,3}$. Alternatively, externally modulated lasers have enabled receiver sensitivities as low as $-7.1 \mathrm{dBm}$ at a BER of $3.8 \times 10^{-3}$, but typically incur a $10 \mathrm{~dB}$ modulation loss ${ }^{4}$. Nyquist-shaped subcarrier modulation (N-SCM) has also been proposed ${ }^{5,6}$, using 16-ary quadrature amplitude modulation (16-QAM) to achieve $112 \mathrm{~Gb} / \mathrm{s}$ at 28 GBaud per channel. However, these also incur significant modulation losses and, hence, have high $\mathrm{CW}$ power requirements.

In this paper, an alternative transceiver design that uses a split-carrier to improve loss budget is presented. The split-carrier method has been recently used to improve the transmitter signal to noise ratio ${ }^{7}$; in this experiment, shown in Fig. 1 , its ability to reduce loss is showcased. The $\mathrm{CW}$ output is split into a carrier branch, inset (i), and signal branch, inset (ii). When recoupled, the transmitter output, inset (iii), is dominated by the carrier, which only suffers coupling losses. The power preserved in the carrier is transfered to the signal at the receiver through direct detection employing only a single photodiode. Using this method, the loss budget is improved and advanced modulation formats become feasible; significantly reducing the transceiver bandwidth requirements. To demonstrate this, a 16-QAM NSCM signal is used to achieve $112 \mathrm{~Gb} / \mathrm{s}$ with a calculated loss budget of $5.2 \mathrm{~dB}$, including the modulator loss.

\section{Experimental configuration}

A CW laser with an output power of $11 \mathrm{dBm}$ emitting at $1551 \mathrm{~nm}$ was split using a $3 \mathrm{~dB}$ polarisation maintaining coupler (PMC) into an upper carrier path and a lower signal path, as shown in Fig. 1. To maintain coherence between the signal and carrier, a narrow-linewidth laser (approximately $10 \mathrm{kHz}$ ) was used at the transmitter; this would not be required in an integrated device, in which differential path length could be controlled. An IQ modulator with a measured dynamic insertion loss of $26.5 \mathrm{~dB}$ was used to modulate the signal. To emulate a simpler design, the four modulator inputs were driven using the same data using a $92 \mathrm{GS} / \mathrm{s}$ digital-to-analogue converter (DAC), generating a $28 \mathrm{GBd} \mathrm{N}-\mathrm{SCM}$ signal. To investigate the optimum carrier-to-signal power ratio (CSPR), the signal was passed through a CSPR controller, comprising an erbium doped fibre amplifier (EDFA) with a noise figure of $5 \mathrm{~dB}$, followed by an optical bandpass filter and a variable optical attenuator (VOA). It is critical to maintain the relative state of polarisation between the carrier and the signal branch. Again, in an integrated device, the CSPR would be defined by the loss of 


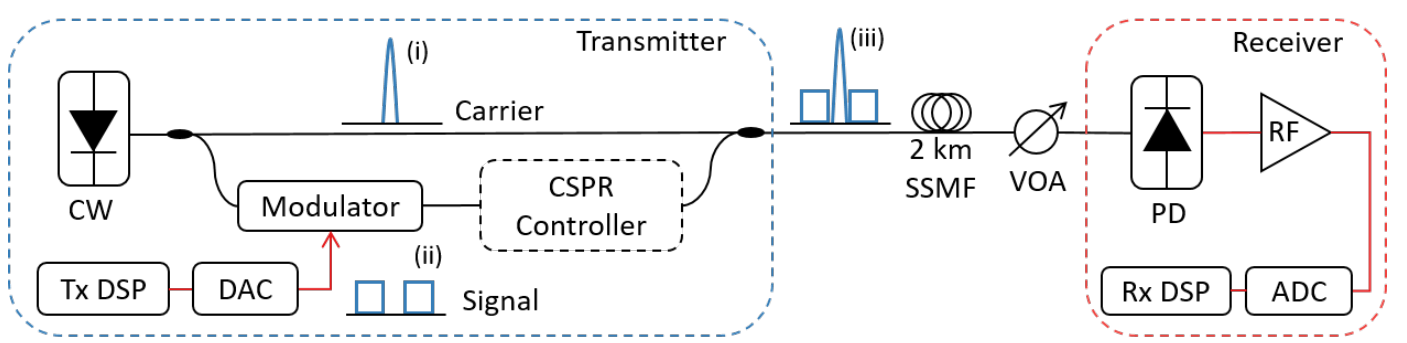

Fig. 1: Experimental setup to investigate the N-SCM split-carrier transceiver. The CSPR controller consists of an EDFA, a bandpass filter and VOA (not required in a final design). The schematic of Fourier spectra are shown in the insets (i)-(iii).

the modulator and the coupling coefficients of the PMCs, with no controller required.

The signal path was then recoupled with the split-carrier using a second $3 \mathrm{~dB}$ PMC, leading a measured total carrier loss of $6.4 \mathrm{~dB}$. The recoupled signal was passed through $2 \mathrm{~km}$ of standard single mode fibre (SSMF) and a VOA prior to detection and RF amplification using a $40 \mathrm{GHz}$ bandwidth photodiode and RF amplifier providing $17 \mathrm{~dB}$ gain, respectively. Finally, the signal was digitised using a $160 \mathrm{GS} / \mathrm{s}$ analogue-todigital converter (ADC) before the offline digital signal processing (DSP) was performed.

The DSP used to generate the N-SCM waveforms is shown in Fig. 2(a). A $2^{16}$ De Bruijin sequence was mapped to 16-QAM symbols before being shaped using a Nyquist root raised cosine (RRC) filter with a roll off factor of 0.05 . The baseband signal was digitally upconverted using a subcarrier with a frequency of $15.4 \mathrm{GHz}$, leading a subcycle ratio of 0.55 . The receiver-side DSP is shown in Fig. 2(b). Following normalisation, the received signal was digitally downconverted using a second $15.4 \mathrm{GHz}$ clock tone. The baseband signal was passed through a RRC filter and resampled to two samples per symbol. An 11-tap equaliser was used to remove inter symbol interference and recover the symbol samples. Finally, the 16-QAM symbols were demapped and passed to a BER estimator.

(a)
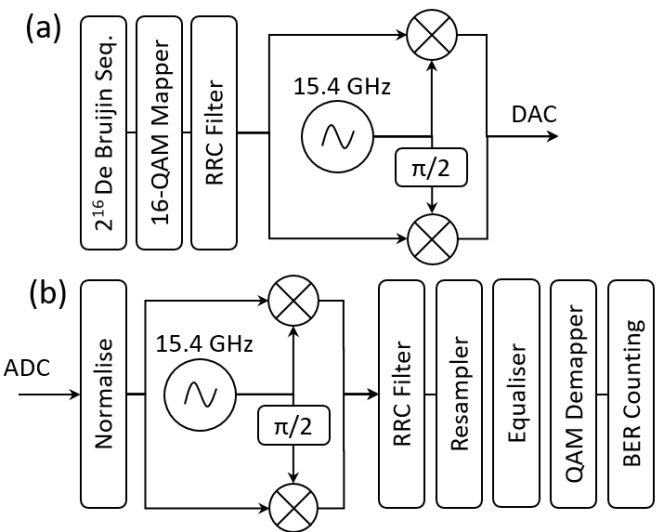

Fig. 2: DSP performed at the (a) transmitter and (b) receiver. A digital clock tone is used to up- and down-convert the data.

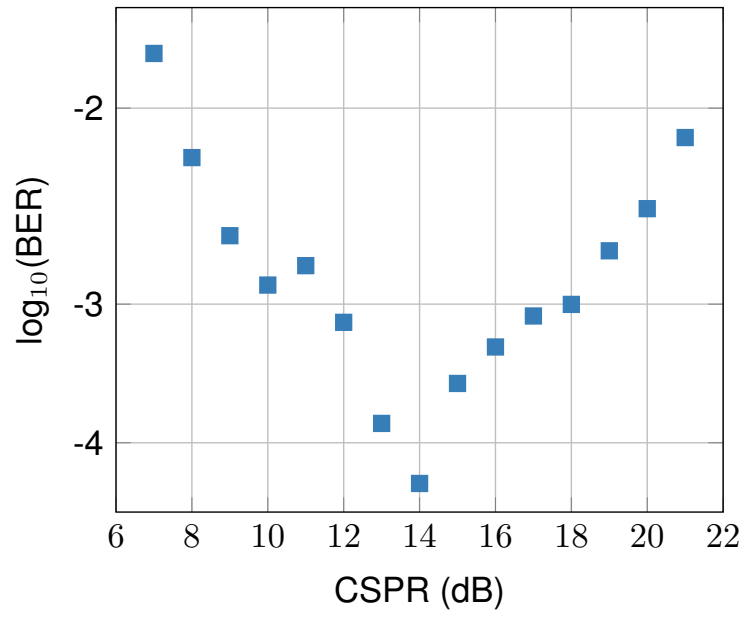

Fig. 3: Variation in system performance for a fixed received power of $-1 \mathrm{dBm}$ when varying carrier to signal power ratio.

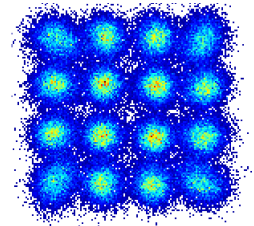

(a)

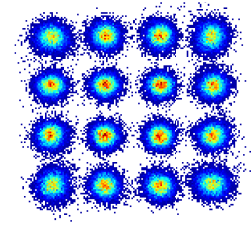

(b)

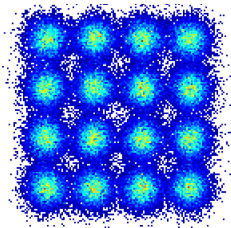

(c)
Fig. 4: Received 16-QAM N-SCM constellations at a received power of $-1 \mathrm{dBm}$ at a CSPR of (a) 8, (b) 14, and (c) $21 \mathrm{~dB}$.

\section{Results}

To determine the optimum PMC coefficient for the split-carrier, the CSPR controller was used to vary the CSPR and back-to-back (BTB) BER measurements taken at a fixed received power of $-1 \mathrm{dBm}$. The results are shown in Fig. 3, in which the optimum CSPR is found to be $14 \mathrm{~dB}$. The effect of this variation on the received 16-QAM constellation can be observed in Fig. 4. Note that in Fig. 4(a), a low CSPR causes amplitude distortions on the outermost symbols due to their high amplitude, shifting the DC bias of the carrier which effectively draws the carrier towards the signal.

To achieve a split-carrier transceiver with a CSPR of $14 \mathrm{~dB}$, the ideal carrier-signal PMC coefficient is 60:40. Assuming both this split ratio, and a typical Mach-Zehnder modulator loss of $\sim 10 \mathrm{~dB}$, then creating the split-carrier scheme establishes a CSPR of $13.5 \mathrm{~dB}$. Crucially, this efficient configuration leads to only $4.4 \mathrm{~dB}$ of cou- 


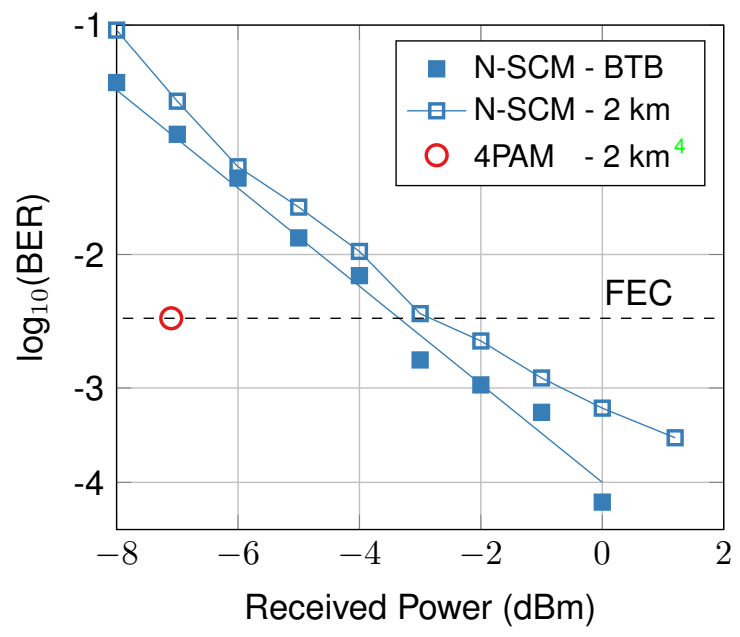

Fig. 5: Performance of the $112 \mathrm{~Gb} / \mathrm{s} \mathrm{N}-\mathrm{SCM}$ split-carrier system. A $112 \mathrm{~Gb} / \mathrm{s}$ 4PAM result is included for comparison.

pling loss for the carrier. For the remainder of this paper, the use of 60:40 PMCs is assumed such that the power lost over the split-carrier is $4.8 \mathrm{~dB}$, with an extra $0.4 \mathrm{~dB}$ added to account for the measured excess coupler losses.

For the transmission experiment, the CSPR was set to $14 \mathrm{~dB}$ and the power received at the photodiode was varied. BTB and transmission over $2 \mathrm{~km}$ of SSMF performance was evaluated and the results are shown in Fig. 5. Applying heuristic fits to the data, a hard-decision forward error correction (FEC) at a BER of $3.8 \times 10^{-3}$ is achieved at a received power of $-3.3 \mathrm{dBm}$ (BTB case) and $-2.7 \mathrm{dBm}$ (after $2 \mathrm{~km}$ ).

The red ring marker in Fig. 5 corresponds to the achieved received power at the FEC threshold after $2 \mathrm{~km}$ of SSMF for a 56 GBd 4PAM signal reported in ${ }^{4}$. It offers $4.4 \mathrm{~dB}$ higher sensitivity compared to the split-carrier reported here. However, it is important to consider this in terms of overall system requirements. Using Eq. (1), the data in Fig. 5 is re-expressed in terms of required CW power, where the $P_{\text {mod,loss }}$ of $10 \mathrm{~dB}$ reported in ${ }^{4}$ is used, while the split-carrier results adopt the optimum $4.8 \mathrm{~dB}$ loss discussed above. A $P_{\text {fibre,loss }}$ of $0.4 \mathrm{~dB}$ is assumed for both cases. The result is seen in Fig. 6. This shows that despite the lower sensitivity, the power-efficient modulation scheme outperforms the 56 GBaud 4PAM result by $0.8 \mathrm{~dB}$ in terms of $\mathrm{CW}$ power requirements. Therefore, the split-carrier N-SCM proofof-concept presented here achieves $112 \mathrm{~Gb} / \mathrm{s}$ with a higher loss budget than the 56 GBaud 4PAM technique reported in ${ }^{4}$, but with reduced bandwidth requirements.

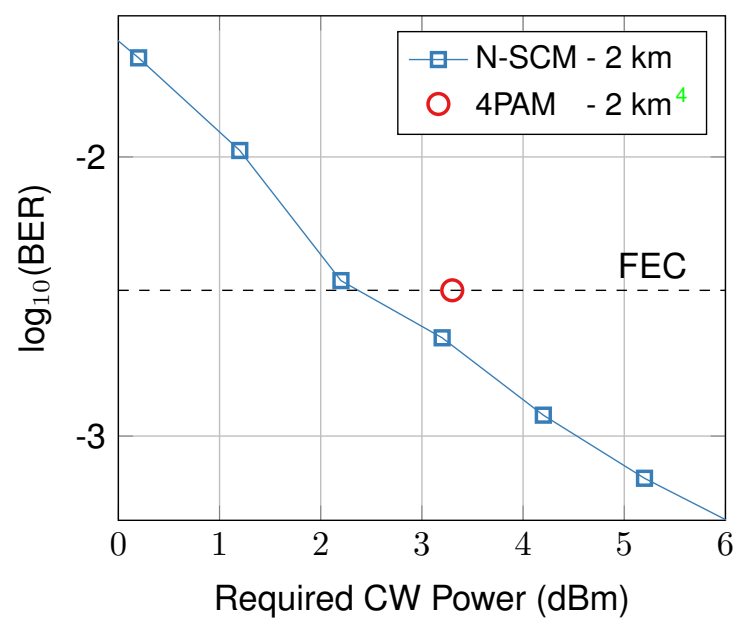

Fig. 6: CW output power required for $112 \mathrm{~Gb} / \mathrm{s}$ over $2 \mathrm{~km}$ using the N-SCM split-carrier system and 4PAM.

\section{Conclusions}

A proof-of-concept split-carrier transmission method envisaged for $400 \mathrm{G}$ intra-datacentre connections is proposed to improve loss budget. The results indicate that optimised coupling coefficients allow a laser-to-photodiode loss budget (over $2 \mathrm{~km}$ ) of $5.2 \mathrm{~dB}$. This was found to outperform a recent, high-sensitivity $112 \mathrm{~Gb} / \mathrm{s}$ 4PAM result by $0.8 \mathrm{~dB}$. Although it is expected that transmitter pre-emphasis and accurate path matching would both improve the receiver sensitivity, the results demonstrate the feasibility of the split-carrier method to reduce the required CW power of externally modulated transmitters.

\section{Acknowledgements}

This work was supported by Microsoft Research through its PhD Scholarship Programme.

\section{References}

[1] IEEE, "P802.3bs 400 Gigabit Ethernet Task Force,", Jul, 15, 2017. [Online] http://www.ieee802.org/3/bs/

[2] Y. Gao et al., "112 Gb/s PAM-4 Using a Directly Modulated Laser with Linear Pre-Compensation and Nonlinear PostCompensation," Proc. ECOC, M.2.C.2, Düsseldorf (2016).

[3] Y. Matsui et al., "55 GHz Bandwidth Distributed Reflector Laser," J. Lightw. Tech., Vol. 35, no. 3, p. 397-403 (2017).

[4] M. Mestre et al., "Compact InP-Based DFB-EAM Enabling PAM-4 112 Gb/s Transmission Over 2 km," J. Lightw. Technol., Vol. 34, no. 7, p. 1572-1578 (2016).

[5] Z. Li et al., "Digital linearization of direct-detection transceivers for spectrally-efficient $100 \mathrm{~Gb} / \mathrm{s} / \lambda \mathrm{WDM}$ metro networking," J. Lightw. Technol., Vol. 36, no. 1, p. 27-36 (2018).

[6] K. Zhong et al., "Transmission of $112 \mathrm{Gbit} / \mathrm{s}$ Single Polarization Half-Cycle 16QAM Nyquist-SCM with 25Gbps EML and Direct Detection," Proc. ECOC, P.4.9, Valencia (2015).

[7] Z. Liu et al.,"448-Gb/s PAM4 Transmission Over 300-km SMF-28 Without Dispersion Compensation Fiber" Proc. OFC, W1J.6, San Diego (2018). 